Raja, Rajiv G.

\section{Quantitation of cDNA expression microarrays for DNA repair and damage response genes}

\author{
R. Raja ${ }^{1,2}$, L. Mascio Kegelmeyer ${ }^{1}$, L. Tomascik-Cheeseman ${ }^{1}$, \\ P. Van Hummelen, F. Marchetti \& A. Wyrobek ${ }^{1}$
}

${ }^{1}$ Biology and Biotechnology Program, Lawrence Livermore National Laboratory, Livermore, California 94550, USA

${ }^{2}$ Reproductive Genetics Division, University of California at San Francisco, San Francisco, California 94143, USA

The analysis of normal and induced variation in gene expression is important for understanding the molecular mechanism of disease and the effects of environmental stress. The advent of microarray technology has provided the means to study expression of large numbers of genes in parallel. In our study, we present a gene expression study, the array development methodology, implementation of custom software for quantifying expression ratios and the expression findings. Our gene expression study employs cDNA from genes of interest (called targets). These are immobilized onto a solid silicon support using an amino modification chemistry which resists strong washing and hybridization procedures. Pools of mRNA from tissues of interest (called probes) are labeled using multiple fluorochromes, hybridized to the array, and the relative amount of fluorescence at each spot is used as a measure of the difference in gene expression. Fluorescence probe labeling strategies were validated for specificity, sensitivity and reproducibility using FITC and Cy3. Multi-color labeling is advantageous for comparing the relative abundance of mRNA from two biological samples within the same array. For probe concentrations ranging from 20 nanograms to 10 picograms, we have demonstrated linear responses for up to 1000 -fold differences in fluorescence intensities. Custom image processing software performs automated background subtraction, grid detection, spot detection and quantitation. Custom perl scripts are used to ratio and normalise data resulting from the image analysis. First, synthetic images were used to test the accuracy of the analysis, and then a known dilution series was prepared and arrayed onto slides to test the entire system.

These tested methods were finally applied to examine the biological significance of expression variation of DNA repair genes in different tissues. We are using cDNA microarrays containing genes involved in double strand break repair, nucleotide excision repair, base excision repair, direct reversal of damage, mismatch repair, cell cycle regulation, apoptosis and damage response. We have identified several differentially expressed genes that may be important for damage response and meiosis.

\section{Ren, Bing}

\section{A yeast genome expression monitoring database}

\section{Bing Ren, Peter Young \& Richard A. Young}

Whitehead Institute for Biomedical Research, 9 Cambridge Center, Cambridge, M assachusetts 02142, USA

The advance in genome sequencing and high-density DNA microarray technologies is transforming the field of biology into an information-processing science. Analysis and sharing of large volumes of data call for new computational tools that few envisioned a few years ago. Of central importance is a database that, at the minimum, facilitates collection, storage and retrieval of biological data. We have designed and developed a database to store and process data obtained from yeast genome monitoring experiments. This database relies on an Oracle database management system running on a Windows NT workstation. We have also developed web-based applications that provide a flexible and friendly interface to the database. Thus, using a web browser, a researcher can upload raw data from microarray experiments to the database, perform various types of data analysis, and more importantly, store the results back into the database to allow higher levels of data analysis. The features of the database also include graphical viewing of gene expression data, clustering analysis, functional categorisation of genes and a search engine that allows user to quickly find and download the expression information for selected genes in selected sets of samples.

Richards, Sue

\section{Optimisation of a dedicated microarray system}

\author{
Sarah Stephens, David Elverd, Keith Haslam \& Sue Richards \\ Genetix Ltd., 63-69 Somerford Road, Christchurch, Dorset BH23 3Q A, UK
}

Microarraying is rapidly becoming the method of choice for high throughput gene expression analysis. Using this relatively simple technique of arraying cDNA's or oligos at high density onto glass slides, it is now possible to analyse thousands of genes in a massively parallel manner. Here we describe some of the parameters that have to be optimised in order to achieve good quality, meaningful microarray data. These parameters include various slide substrates, pin design, hybridisation systems and reading of arrayed slides.

Ringwald, Martin

\section{Gene expression database for the laboratory mouse}

\author{
M. Ringwald, J.T. Eppig, J. Kadin \& J. Richardson \\ The Jackson Laboratory, Bar Harbor, M aine 04609, USA
}

The laboratory mouse serves as a pivotal animal model in gene expression and function studies, because it is closely related to the human and because tissues from many different mouse strains and mutants are readily available for detailed expression analysis. Array-based methods will provide a tremendous amount of expression information. In order to take full advantage of these data, we must be able to analyse them in a broader biological context.To address these issues and to cope with the complexity of gene expression information for the mouse in general, we developed the Gene Expression Database (GXD). GXD is designed as an open-ended system that can integrate many different types of expression data, such as RNA in situ hybridisation and immunohistochemistry data, Northern and Western blot data, RT-PCR data, cDNA data and microarray data. Thus, as data accumulate, GXD can provide increasingly complete information about what transcripts and proteins are produced by what genes; where, when and in what amounts these gene products are expressed; and how their expression is affected in different mouse strains and mutants. Through collaborative efforts with the MRC Human Genetics Unit and the University in Edinburgh, UK ${ }^{1}$, we have established a comprehensive hierarchically structured dictionary of anatomical terms for the mouse. The dictionary is used to annotate expression patterns in standardised ways and will facilitate an integrated description of expression, anatomy and phenotype/disease data. GXD is available at http://www.informatics.jax.org/. It is integrated with the Mouse Genome Database (MGD) to enable a combined analysis of genotype, expression and phenotype data. In collaboration with Flybase, the Saccharomyces Genome Database and MGD we are building shared controlled vocabularies to describe biological processes, and cellular function and location of gene products. These classification schemes and the associated data curation 